\title{
Hydrodynamic drag-force measurement and slip length on microstructured surfaces
}

\author{
A. Maali, ${ }^{1, *}$ Y. Pan, ${ }^{2,3}$ B. Bhushan, ${ }^{2, \dagger}$ and E. Charlaix ${ }^{4}$ \\ ${ }^{1}$ Laboratoire Ondes et Matière d'Aquitaine (LOMA), Université Bordeaux I, 351 cours de la Liberation, F-33405 Talence, France \\ ${ }^{2}$ Nanoprobe Laboratory for Bio- \& Nanotechnology and Biomimetics $\left(N L B^{2}\right)$, The Ohio State University, \\ 201 W. 19th Avenue, Columbus, OH 43210-1142, USA \\ ${ }^{3}$ Mechanical Engineering, Harbin Institute of Technology, Harbin, 150001, P.R. China \\ ${ }^{4}$ Laboratoire Interdisciplinaire de Physique (LIPhy), Université Joseph Fourier, F-38402 Saint Martin d' Hères, France
}

(Received 19 August 2011; revised manuscript received 21 December 2011; published 14 June 2012)

\begin{abstract}
In this paper, a drainage experiment of water between a borosilicate sphere and a microstructured surface constituted by regularly spaced pillars is presented. The microstructured surface has two parts: on one part the liquid forms a Cassie interface and on the second it forms a Wenzel interface. The measured hydrodynamic drag force is larger on the Cassie part compared to the Wenzel part. Furthermore, for the Cassie part, from the hydrodynamic drag force measurements on a pillar and between pillars the corresponding local slip lengths have been extracted. The area average slip length on the surface is in agreement with the value expected by Philip's equation.
\end{abstract}

DOI: 10.1103/PhysRevE.85.066310

PACS number(s): 47.61.-k, 46.55.+d, 07.79.Lh, 61.48.De

\section{INTRODUCTION}

Liquid flow close to a solid surface has received much attention recently and it has been studied theoretically and experimentally by several groups [1-26]. Using newly developed techniques, it is actually possible to drive and probe the flow at the micro and nanoscales. For a smooth hydrophobic surface, there is an agreement that the flow should be described by a partial slip boundary condition. The amount of fluid slippage is commonly represented by the slip length $b$, which is typically on the order of tens of nanometers on smooth homogeneous surfaces. Very large slip lengths were reported on microstructured surfaces [13-18]. A large slip length indicates that the liquid flow between confining surfaces experiences a lower friction, and a solid moving in a liquid experiences a lower drag force. Therefore, creating surfaces that increase slip length becomes a topic of great interest in micro and nanofluidics devices.

Ou et al. $[13,14]$ combined the microparticle image velocity ( $\mu$-PIV) and pressure drop versus flow rate measurements to study the flow through a channel structured with microridges. They showed a large hydrodynamic drag force reduction for the fluid flow, and also that the slip along the shear free air-interface supported between the hydrophobic microridges is primarily responsible for the hydrodynamic drag force reduction. Hydrodynamic drag force reduction is much more important as the fraction of the area of the air-interface becomes larger compared to the solid area. For a flat composite interface the magnitude of slippage is proportional to the periodicity of the gaseous patterns [13-23].

Recently the effect of the edges of the holes or stripes of the nanostructures on liquid flow close to the interface has been investigated. These edges induce curvatures of the airsolid interface and thus increase the friction (drag) instead of reducing it. The demonstration of this effect was shown using a dynamic surface force apparatus (SFA) $[24,25]$. It was shown

\footnotetext{
*Corresponding author: a.maali@loma.u-bordeaux1.fr †Bhushan.2@osu.edu
}

that the curvature of the liquid gas menisci has a dramatic influence on the boundary condition, turning it from slippery to sticky. The trapped bubbles at the holes of the nanostructure act as an anti-lubricant and promote high friction [24-26].

In this paper a colloidal probe atomic force microscopy (AFM) is used to measure hydrodynamic drag force and slip length on a microstructured surface.

\section{SURFACE PREPARATION AND CHARACTERIZATION}

The surfaces used in this experiment are made from silicon and consist of pillars of 5.1 microns in diameter $(2 a)$ spaced by 7.4 microns (pitch $L$ ). The height of the pillars is 15 microns. The flat silicon surface has a contact angle of $25^{\circ}$, which is hydrophilic. The surface is covered with a layer of tetrahydroperfluorodecyltrichlorosilane $\left(\mathrm{PF}_{3}\right)$, which makes it hydrophobic [27]. Optical measurements show that a water droplet forms a contact angle of $137^{\circ}$ on the surface. On one part of the surface the contact angle was reduced by applying a voltage of $20 \mathrm{~V}$ between the liquid and the substrate (irreversible electrowetting process). This process induces an irreversible transition from the Cassie state with air pocket formation to the Wenzel state, which means that the water penetrates between the pillars, and the measured contact angle was $117^{\circ}$. Using this process a sample was created divided in two parts; in the first one the liquid at the interface forms a Cassie state, and in the second part the liquid at the interface forms a Wenzel state [28]. Such a sample offers the advantage of switching during the experiment from the Cassie interface to the Wenzel interface just by translating the sample horizontally at two locations (with and without electric field applied) without changing samples.

Before performing the drainage experiment, the surfaces are imaged in liquid using an AFM (Bruker, Dimension 3000) equipped with a liquid cell that allows tapping mode imaging in liquid. A rectangular cantilever (ORC8, Veeco) having nominal values of $0.3-\mathrm{N} / \mathrm{m}$ stiffness and $10-\mathrm{nm}$ tip radius, respectively, has been used. The obtained images are shown in Fig. 1. The set-point imaging amplitude was chosen to be close to the free amplitude to minimize the applied force to the 


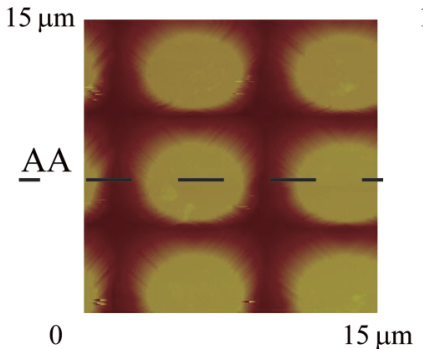

(a)

Horizontal section - AA

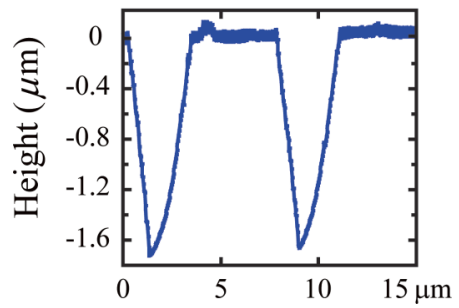

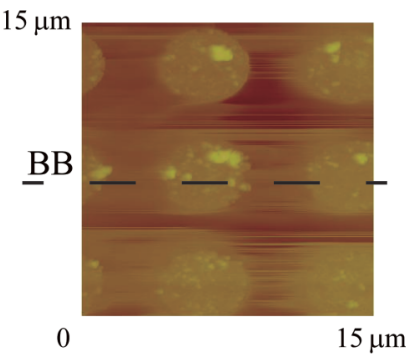

(b)

Horizontal section - BB

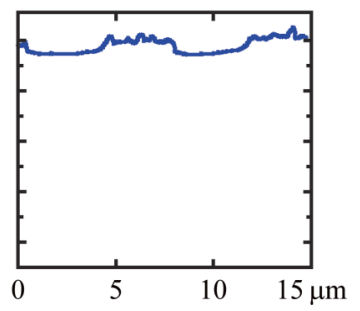

FIG. 1. (Color online) AFM images of the nanostructured surfaces in water. (a) Wenzel part (electric field applied) and (b) Cassie part. Note here that the height measured in (a) does not match the pillar height of $15 \mu \mathrm{m}$, instead it is $1.6 \mu \mathrm{m}$. This is because the height of the tip on the cantilever is $2 \mu \mathrm{m}$, which limits the depth the tip can penetrate and the measurement reflects the tip shape.

sample during imaging. The figure shows the images obtained in the Wenzel and Cassie parts and horizontal sections in both images. It was found that the water penetrates between the pillars. However, the image of the Cassie part of the surface shows that water does not penetrate between the pillars, which lead to the trapping of gas pockets between the liquid and solid.

\section{EXPERIMENTAL RESULTS AND DISCUSSION}

To measure hydrodynamic drag forces, drainage experiments were performed using the AFM in contact mode. The hydrodynamic drag force is given by the deflection of the cantilever as the sphere attached to the cantilever approaches the sample with a constant velocity. A spherical borosilicate particle (GL0186B/45-53, MO-Sci Corporation) with a diameter of $52.5 \mu \mathrm{m}$ was used. The benefits of using such a large particle is to minimize the hydrodynamic drag force due to the cantilever itself and to increase the hydrodynamic drag force due to the sphere. Also with such a large sphere, a large separation distance of up to $D=2000 \mathrm{~nm}$ can be investigated while still remaining in the lubrication approximation $(D \ll R)$. The sphere was glued to the end of a silicon nitride rectangular cantilever (ORC8, Bruker) using epoxy (Araldite, Bostik, Coubert). To analyse the data we have followed the procedure by Zhu et al. $[10,11]$ and Honig et al. [12]. Especially to remove the contribution of friction in the conversion of the photodiode voltage to deflection (nm), we have used both extended and retracted curves.

The stiffness $k$ of the cantilever with a sphere attached was determined in situ using a flat silicon surface as a substrate [Fig. 2(a)].

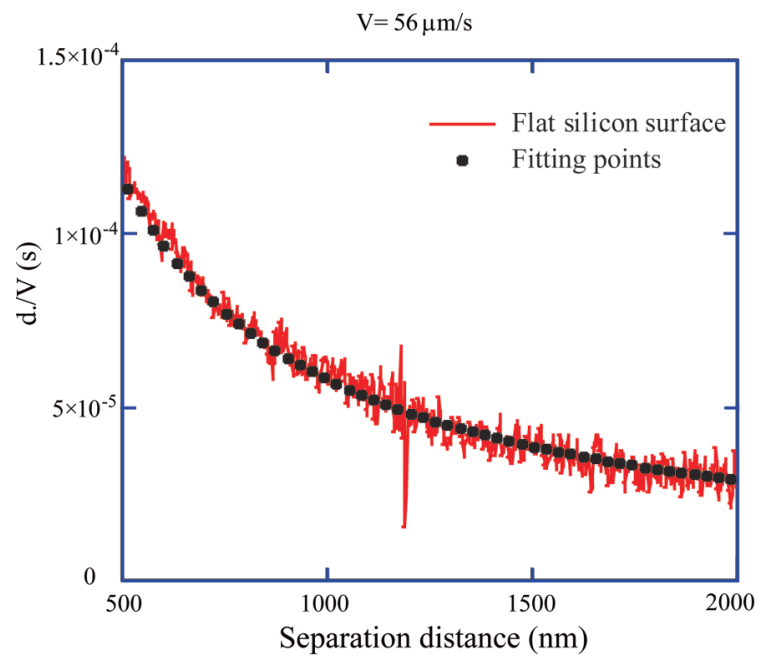

(a)

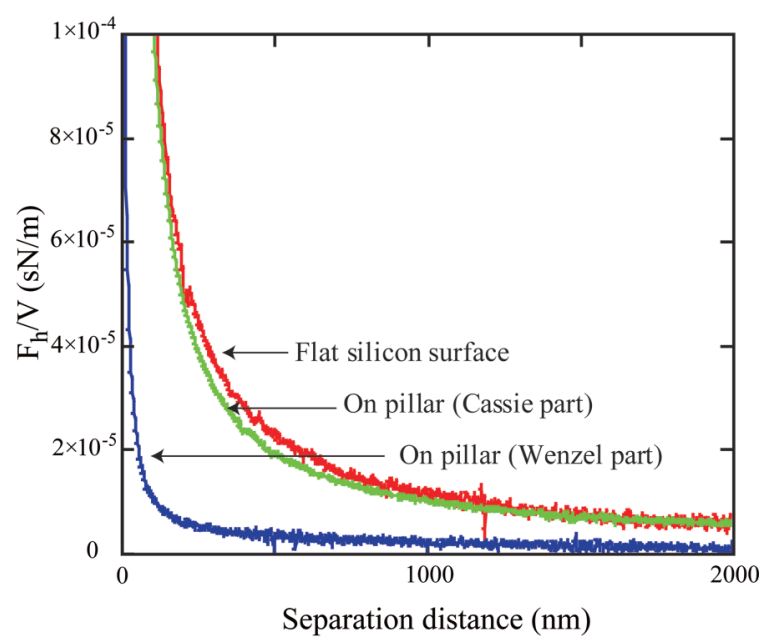

(b)

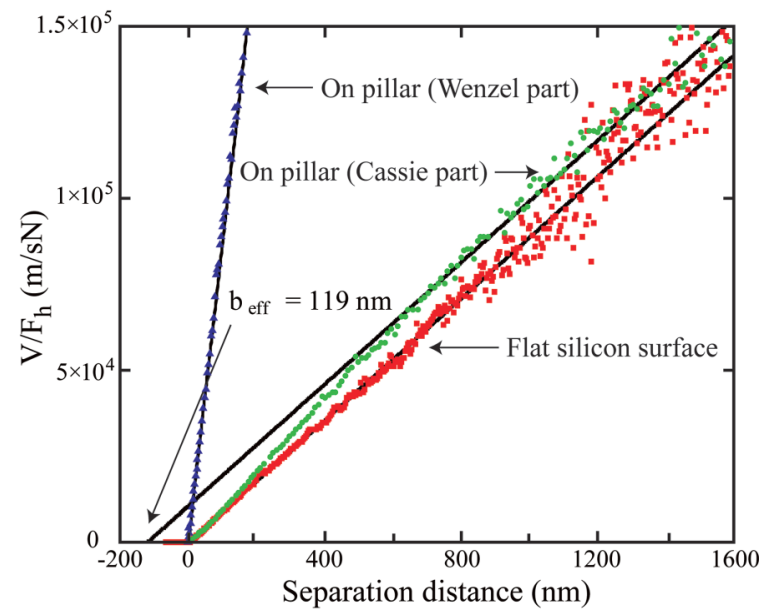

(c)

FIG. 2. (Color online) (a) The $d / V$ for the flat silicon surface and the fitting points using (1). From the fit $\frac{6 \pi \eta R^{2}}{k}=0.058 \mathrm{~nm} / \mathrm{s}$ is obtained and thus a stiffness value of $k=0.19 \mathrm{~N} / \mathrm{m}$, (b) the measured hydrodynamic drag force divided by velocity as a function of separation distance for the different surfaces, and (c) velocity divided by the hydrodynamic drag force $V / F_{h}$ for the measurement on the flat silicon surface and on a pillar for both the Cassie part and the Wenzel part of the microstructured surface. 
The deflection of the cantilever $(d)$ is related to the force $\left(F_{h}\right)$ by

$$
\frac{d}{V}=\frac{F_{h}}{k V}=\frac{6 \pi \eta R^{2}}{k D},
$$

where $R$ and $V$ are the radius and the velocity of the approaches, $k$ is the cantilever stiffness, and $\eta$ is the dynamic viscosity of the fluid. The velocity of the approaches was obtained from the time derivatives of distance [12]. From the fit of the data [Fig. 2(a)] in the long-range distance (500-2000 nm) $\frac{6 \pi \eta R^{2}}{k}=0.058 \mathrm{~nm} / \mathrm{s}$ is obtained and thus a stiffness value of $k \stackrel{k}{=} 0.19 \mathrm{~N} / \mathrm{m}$.

First the sphere is centered on the center of a given pillar of the surface, and then the measurements of the hydrodynamic drag force as a function of the distance between the sphere and the surface of the pillar are performed. On each surface the measurement is made on several pillars to avoid problems of inhomogeneities and surface roughness of the pillars. The measured force on the structured surface is shown in Fig. 2(b). The measured force on the Wenzel part of the microstructured surface is smaller than the force on the flat silicon surface. This is easily understood by the fact that some of the flow occurs between pillars which are very high, and thus a lower hydrodynamic drag force is exerted on the sphere. For the Cassie part of this hydrophobic surface the force is roughly equal to the force measured on the flat silicon surface. This observation may seem strange. A large water slip on a hydrophobic surface is expected, thereby reducing the hydrodynamic drag force. In this case, the hydrodynamic drag force measured when the sphere is centered on a pillar of the Cassie part of the microstructured surface is not very small compared to the measured force on the silicon surface, which suggests a small slip.

To accurately see the behavior of the water drainage and to extract the slip length $b$, it is more convenient to present the data in the form $V / F_{h}$ as was illustrated by Cottin-Bizonne et al. [8]. In the limit of large separation distance $(D>b)$

$$
V / F_{h}=\frac{D+b_{\mathrm{eff}}}{6 \pi \eta R^{2}},
$$

where $b_{\text {eff }}$ is the effective slip length. The advantage to presenting the data in the form of $V / F_{h}$ is that the slip length appears as the position where the linear extrapolation of the curve $V / F_{h}$ intercepts the distance axis. $V / F_{h}$ as a function of separation distance is plotted in Fig. 2(c). For the measurement on a pillar on the Cassie part, the data $V / F_{h}$ for larger distances have the same slope on the silicon surface $\frac{1}{6 \pi \eta R^{2}} \approx 88.6 \mathrm{nN}^{-1} \mathrm{~s}^{-1}$. A linear fitting for a distance between 400 to $1400 \mathrm{~nm}$ uses the slope extracted from the measurement on the flat silicon surface as shown in Fig. 2(c). In this range of distance, the hydrodynamic radius $\sqrt{2 R D}$ varies from 5 to $8 \mu \mathrm{m}$, which means that the area probed by the flow is at least four times as large as the pillar surface and reaches the unit cell of the square lattice. The linear extrapolation of the fitting line intercepts the distance axis at $-119 \mathrm{~nm}$. Based on (2) we interpret this value as an averaged slip length $b_{\text {eff }}=$ $119 \mathrm{~nm}$, describing the flow on a pillar, probed on one lattice unit cell.
For the data on the Wenzel part of the microstructured surface the slope is completely different from that of the data on the flat silicon surface. Only the data for distances of less than $200 \mathrm{~nm}$ are presented because for larger distances the hydrodynamic drag force is very small, as is shown in Fig. 2(b). For larger distances any small error in the determination of the Stokes term (contribution of the cantilever beam to the hydrodynamic drag force) induces a large variation on the value of $V / F_{h}$. For the data on the Wenzel part presented in Fig. 2(c), $V / F_{h}$ has a linear variation versus the distance without slip. The linear fitted curves give a slope of $891 \mathrm{nN}^{-1} \mathrm{~s}^{-1}$. The value of this slope is ten times larger than the slope on the silicon surface, and it is roughly equal to the ratio between the radius of the sphere and the radius of the pillar $R / a=10.4$. This result is different from what can be expected from analytical calculations using the lubrication approximation. For very small distances compared to the radius of the pillar $(a=2.52 \mu \mathrm{m})$ the analytical calculation suggests a slope on the pillar of the Wenzel surface equal to the slope on the flat silicon surface, $\frac{1}{6 \pi \eta R^{2}} \approx 88.6 \mathrm{nN}^{-1} \mathrm{~s}^{-1}$. However, the data show that the slope of $V / F_{h}$ on a pillar of the Wenzel part is ten-fold larger.

Next, the liquid slip is investigated on the Cassie part of the microstructured surface. To get a true picture of the liquid flow on the surface, the flow between the pillars must be probed. The colloidal probes of an AFM offer this opportunity since it allows the centering of the sphere between four pillars and then measures the local hydrodynamic drag force. The measured data are shown in Fig. 3(a). The data on the Cassie pillar and on the flat silicon surface are also reported to compare them. Between four pillars the edge of the sphere penetrates between them by an amount of $\frac{(\sqrt{2} L-2 a)^{2}}{8 R} \approx 137 \mathrm{~nm}$. Thus for the measurements between four pillars the hard contact position is $137 \mathrm{~nm}$ below the pillars' surface. In our analysis of the data we choose the pillars' surface as the reference surface, which is consistent if we want to compare our measurements with the theoretical models that calculate a macroscopic effective slip length on such a surface where the pillars' surface is taken as the reference surface.

In the inset is shown hydrodynamic drag force $F_{h} / V$ for the Cassie part of the microstructured surface for different velocities $V$ of approaches 32, 56, and $112 \mu \mathrm{m} / \mathrm{s}$. The force is independent of the velocity, which means that the liquidgas interface is not disturbed by the pressure induced by the approaching sphere. Furthermore, it was found that there is no capillary attraction of the interface to the sphere (data not shown). This is in agreement with the image presented in Fig. 1(b), which shows that the interface is underneath the reference plane formed by the pillars' surface.

The value of the hydrodynamic drag force depends on the position where it is measured on the Cassie part of the microstructured surface. The value of the hydrodynamic drag force is lower when measured between pillars compared to when it is measured on a pillar itself. This can be explained by considering the streamline of the flow [see Fig. 3(c)]. When the sphere is centered on the pillar, the liquid squeezed out by the approaching sphere will flow on a modulated topographic interface. For the measurements between four pillars, a part of the liquid squeezed out will flow in a straight line between the pillars over the liquid-gas interface. This part of the flow 


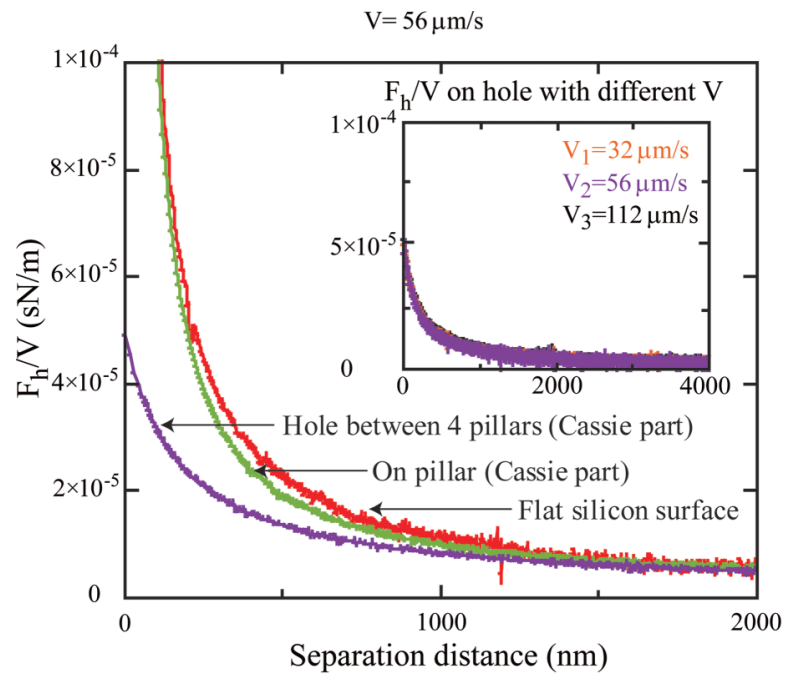

(a)

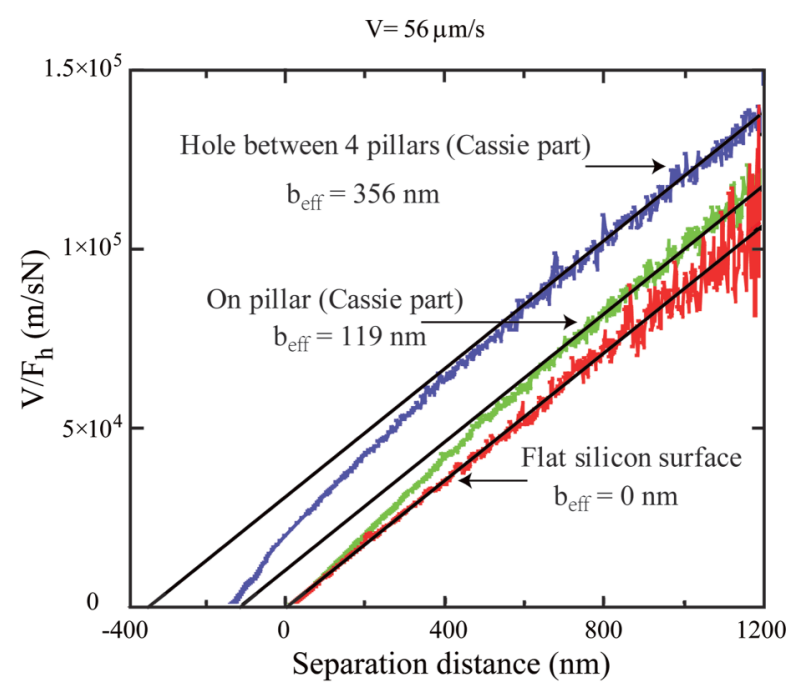

(b)

Flow Streamlines

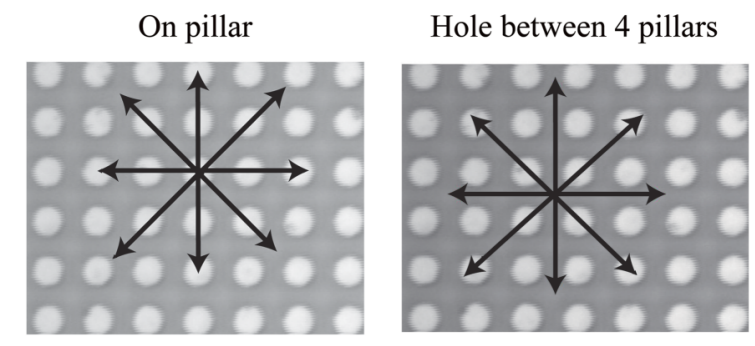

(c)

FIG. 3. (Color online) (a) The measured hydrodynamic drag force on flat silicon and on the Cassie part of the surface when the sphere is centered between four pillars and on a pillar of the microstructured surface. The data on the silicon surface are reported for comparison. In the inset $F_{h} / V$ is shown between four pillars for different velocities $V$ of approaches 32, 56, and $112 \mu \mathrm{m} / \mathrm{s}$. (b) Velocity divided by the hydrodynamic drag force $V / F_{h}$ as a function of separation distance. The solid lines are linear extrapolations using the same slope for the three curves. The fitted values provide slip length $b_{\text {eff }}$ are indicated on each curve. (c) The flow streamlines close to the surface when the sphere is centered on a pillar and between four pillars. occurs with less friction, and thus the total hydrodynamic drag force exerted on the sphere is reduced.

To calculate slip lengths, the measured data are plotted as $V / F_{h}$ versus separation distance in Fig. 3(b). The plots obtained on the Cassie part of the microstructured surface (on pillars and between pillars) have the same slope as on the silicon surface. Furthermore, the measurements show that the flow when the sphere is centered between four pillars occurs with a larger slip compared to the flow when the sphere is centered on a pillar. The extracted slip length from the fitting of the data gives $b_{\text {eff }}^{1}=119 \pm 30 \mathrm{~nm}$ and $b_{\text {eff }}^{2}=356 \pm 30 \mathrm{~nm}$, respectively, on pillars and between four pillars, probed on the same area.

How can one connect these measurements to what is measured with other techniques? With the colloidal probe AFM we have access to semilocal values of the slip length on a heterogeneous surface, in contrast to other techniques that probe an average flow. Based on the fact that the "pillarcentered" and the "between-four-pillars" positions correspond to the extreme of the drag force at a given distance, we expect that the effective slip length at large distance is given by $b_{\mathrm{eff}}^{a v}=$ $\phi_{s} b_{\text {eff }}^{1}+\left(1-\phi_{s}\right) b_{\text {eff }}^{2}$, where $\phi_{s}$ is the solid fraction given as $\pi a^{2} / L^{2}=0.37$. For this value of $\phi_{s}, b_{\text {eff }}^{a v}=268 \pm 30 \mathrm{~nm}$.

This average slip length can be compared to theoretical models for a periodically patterned surface. The effective slip length on a two-dimensional array of pillars separated by a flat, free surface has been calculated by Davis and Lauga [23], generalizing the result of Bocquet et al. [22] $b_{\text {eff }}=L\left(\frac{3}{16} \sqrt{\frac{\pi}{\phi_{s}}}-\frac{3}{2 \pi} \ln (1+\sqrt{2})\right)$. The corresponding value for our micro-structured surface is $b_{\text {eff }}=926 \mathrm{~nm}$. This value overestimates by far our measurements. The model indeed does not take into account the curvature of the menisci which increases significantly the interfacial friction. A more appropriate model to take into account this three-dimensional (3D) character of the liquid boundary is the Philip's calculation of the slip length on a periodic surface made of grooves oriented parallel to the flow [19]. Indeed due to the lattice symmetry, the 3D undulation of the liquid free surface due to the menisci disappears completely along the lines lying at equal distance of two adjacent pillar rows, whereas it is maximum along the lines lying exactly on the pillar centers. Therefore if we take the grooves' size equal to the separation between two adjacent pillars $(L-2 a=2.3 \mu \mathrm{m})$ the Philip's model underestimates the actual friction on the grooves, but overestimates the friction between the grooves. The area fraction of the liquid-gas interface for this geometry is given by $\phi_{g}=\frac{L-2 a}{L}=0.31$. The value of the slip length given by $[18,19]$ is $b_{\text {eff }}=\frac{-L}{\pi} \ln \left[\cos \left(\frac{\pi}{2} \phi_{g}\right)\right]=291 \mathrm{~nm}$. This calculated value is close to the average slip length measured in this experiment.

\section{CONCLUSION}

A drainage experiment of water between a borosilicate sphere and a microstructured surface constituted by regularly spaced pillars has been presented. The measured hydrodynamic drag force is larger on the Cassie part compared to the Wenzel part. These measurements show the important role played by the shape of the interface on the liquid flow. 
Furthermore, in the Cassie part of the microstructured surface a larger hydrodynamic drag force was measured on the pillar than between pillars, although the area probed by the flow is on order of the lattice unit cell. Corresponding values of semilocal, coarse-grained slip lengths were extracted, the aver- age value of which gives a reliable estimate of the effective slip length. We find that the effective slip length is well described by the Philip's equation for grooves parallel to the flow, which shows that an important consequence of the menisci curvature is to favor the flow between adjacent pillars' rows.
[1] C. L. M. H. Navier, Mem. Acad. Roy. Sci. Inst. Fr. 6, 389 (1823).

[2] N. V. Churaev, V. D. Sobolev, and A. N. Somov, J. Colloid Interface Sci. 97, 574 (1984).

[3] E. T. Watts, J. Krim, and A. Widom, Phys. Rev. B 41, 3466 (1990).

[4] E. Lauga, M. P. Brenner, and H. A. Stone, Microfluidics: The NoSlip Boundary Condition, Volume Handbook of Experimental Fluid Dynamics (Springer, New-York, 2005), Chap. 15.

[5] J. L. Barrat and L. Bocquet, Soft Matter 3, 685 (2007).

[6] L. Bocquet and E. Charlaix, Chem. Soc. Rev. 39, 1073 (2010).

[7] A. Maali and B. Bhushan J. Phys.: Condens. Matter 20, 315201 (2008).

[8] C. Cottin-Bizonne, B. Cross, A. Steinberger, and E. Charlaix, Phys. Rev. Lett. 94, 056102 (2005).

[9] D. Lasne, A. Maali, Y. Amarouchene, L. Cognet, B. Lounis, and H. Kellay, Phys. Rev. Lett. 100, 214502 (2008).

[10] L. Zhu, P. Attard, and C. Neto, Langmuir 27, 6701 (2011).

[11] L. Zhu, P. Attard, and C. Neto, Langmuir 27, 6712 (2011).

[12] C. D. F. Honig and W. A. Ducker, Phys. Rev. Lett. 98, 028305 (2007).

[13] J. Ou, B. Perot, and J. P. Rothstein, Phys. Fluids 16, 4635 (2004).

[14] J. Ou and J. P. Rothstein, Phys. Fluids 17, 103606 (2005).

[15] C. H. Choi and C. J. Kim, Phys. Rev. Lett. 96, 066001 (2006).
[16] P. Joseph, C. Cottin-Bizonne, J. M. Benovi, C. Ybert, C. Journet, P. Tabeling, and L. Bocquet, Phys. Rev. Lett. 97, 156104 (2006).

[17] R. Truesdell, A. Mammoli, P. Vorobieff, F. van Swol, and C. J. Brinker, Phys. Rev. Lett. 97, 044504 (2006).

[18] C. Lee, C. H. Choi, and Chang-JinCJ Kim, Phys. Rev. Lett. 101, 064501 (2008).

[19] J. Philip, Z. Angew. Math. Phys. 23, 353 (1972); 23, 960 (1972).

[20] E. Lauga and H. A. Stone, J. Fluid Mech. 48, 55 (2003).

[21] F. Feuillebois, M. Z. Bazant, and O. I. Vinogradova, Phys. Rev. Lett. 102, 026001 (2009).

[22] C. Ybert, C. Barentin, C. Cottin-Bizonn, P. Joseph, and L. Bocquet, Phys. Fluids 19, 123601 (2007).

[23] A. M. J. Davis and E. Lauga, J. Fluid Mech. 661, 402 (2010).

[24] A. Steinberger, C. Cottin-Bizonne, P. Kleimann, and E. Charlaix, Nat. Mater. 6, 665 (2007).

[25] A. Steinberger, C. Cottin-Bizonne, P. Kleimann, and E. Charlaix, Phys. Rev. Lett. 100, 134501 (2008).

[26] J. Hyvaluoma and J. Harting, J. Phys. Rev. Lett. 100, 246001 (2008).

[27] B. Bhushan and Y. C. Jung, Prog. Mater. Sci. 56, 1 (2011).

[28] M. Nosonovsky and B. Bhushan, Multiscale Dissipative Mechanisms and Hierarchical Surfaces: Friction, Superhydrophobicity, and Biomimetics (Springer-Verlag, Heidelberg, 2008). 